\title{
Discrimination of the rate of filtered impulses
}

\author{
ERVIN R. HAFTER and VIRGINIA M. RICHARDS \\ University of California, Berkeley, California
}

\begin{abstract}
When subjects detect changes in interaural differences of time or level in the envelope of a complex waveform, the accuracy of the detection depends on the stimulus's duration. The change in threshold with duration, in turn, depends on the rate of modulation of the waveform's envelope. As the envelope rate increases beyond about $85 \mathrm{~Hz}$, detectability relies increasingly on information derived from the early portion of the signal. The purpose of the present experiment was to determine whether a similar reliance on onset information might affect the detection of changes in envelope rate per se. The stimuli were trains of high-frequency, filtered clicks that differed only in their click rates. Control conditions included low-frequency noise intended to interfere with nonlinear distortion and a roving carrier technique designed to discourage comparisons between individual harmonic components. Unlike the case for lateralization, ratediscrimination thresholds for click rates as high as $400 \mathrm{~Hz}$ declined with the square root of duration, indicating that listeners derived information from throughout the stimulus. From these data it is suggested that separate channels may be used to convey envelope information for spatial hearing and for the discrimination of pitch.
\end{abstract}

It has long been known that trains of impulses repeated at moderate rates produce a pitch associated with the click rate; the connection between this pitch and the period of the signal has given rise to the name periodicity pitch. The spectrum of such signals concentrates energy at frequencies harmonically related to the fundamental frequency (inverse of the period), and much of the interest in them has stemmed from the fact that their pitch remains strong even when the fundamental itself is absent. This result has led to models that suggest that the listener uses some form of correlational analysis to extract periodicity in either the time (e.g., Schouten, 1940, 1970; Schouten, Ritsma, \& Cardozo, 1962) or the frequency (e.g., Goldstein, 1973; Terhardt, 1974; Wightman, 1973) domain.

The present study was designed to examine one aspect of periodicity coding, the effect of signal duration on the ability to discriminate between two complex signals of different fundamental frequencies. It was motivated by the results of a series of experiments on lateralization, in which the ability to derive information from the envelopes of high-frequency complexes was studied by measuring the detectability of interaural differences of time or intensity in trains of high-frequency clicks. In such cases, the effectiveness of the interaural information tends to be nonuniform over the duration of the train, declining after the stimulus's onset. The intent here was to apply a similar analysis to the discrimination of click rate, that is, the discrimination of periodicity pitch, to determine the course of information accumulation from a complex signal where binaural interaction is not involved.

This work was supported in part by NIH Grant $1-44036$ and in part by UC Chancellor's Patent Grant NS-07787. E. R. Hafter's mailing address is Department of Psychology, 3210 Tolman Hall, University of California, Berkeley, CA 94720 . V. M. Richards's mailing address is Psychoacoustics Lab, Department of Psychology, University of Florida, Gainesville, FL 32611.

\section{Analysis of the Useful Information}

\section{in a Train of Clicks}

In the most common of the binaural experiments, subjects have listened for interaural differences of time (IDTs) in trains of high-frequency (bandpassed at $4 \mathrm{kHz}$ ) clicks (Hafter \& Dye, 1983). Click rate was denoted by its inverse, the interclick interval (ICI), duration by the number of clicks in a train $(n)$, and threshold $\left(\Delta \mathrm{IDT}_{n}\right)$ by the interaural delay necessary to produce a $d^{\prime}$ of 1.0 . One must assume that discrimination in such experiments is limited by an "internal noise" in the domain of the signal, here a temporal jitter added by the nervous system of the observer. Detection theory argues that the standard deviation of the internal noise can be obtained directly from the threshold for a single dichotic click $(n=1)$ since

$$
d^{\prime}=1.0=\frac{\Delta \mathrm{IDT}_{1}}{\delta_{\mathrm{IDT}_{1}}}
$$

Where the train consists of more than one click, if each is equally effective and if the jitter added to the successive samples of the IDT is independent, statistical averaging across the sum of the IDT + jitter should lead to a $\sqrt{ } n$ improvement in performance:

$$
\Delta \mathrm{IDT}_{n}=\frac{\Delta \mathrm{IDT}_{1}}{n^{0.5}} .
$$

For comparisons to data, we have found it useful to use the logarithmic form of this expression, which predicts a straight line with intercept at the log threshold for a single click and slope of -0.5 :

$$
\log \Delta \mathrm{IDT}_{n}=\log \Delta \mathrm{IDT}_{1}-0.5 \log n .
$$

\section{Lateralization and the Effects of Click Rate}

Whereas listeners readily detect IDTs in single transients regardless of frequency content, they cannot do so 
if the signal is an ongoing high-frequency tone (Blauert, 1983) or an amplitude-modulated high-frequency tone with a high rate of modulation (Nuetzel \& Hafter, 1981). This implies that for interaural delay, the initial transient is more informative than the portion that follows. In pursuit of this notion, Hafter and Dye (1983) suggested the use of a "method of subtraction" (Hafter, Buell, \& Richards, in press), whereby differences found between thresholds obtained with two different stimulus durations are attributed to information carried in the extra portion of the longer signal. For these tests, they used click trains of various lengths $(n)$ and examined the effect of increasing the rate (1/ICI). To describe the actual effectiveness of successive clicks, they rewrote Equation 3 in terms of an intervening variable, $N_{n}$, defined as the number of informative neural events evoked by a train of length $n$ :

$$
\log \Delta \mathrm{IDT}_{n}=\log \Delta \mathrm{IDT}_{1}-0.5 \log N_{n} .
$$

Clearly, psychophysics alone cannot substitute for the direct observation of neural activity, but it allows for relative measures through which the effectiveness of one stimulus can be compared with that of another. While Equation 3 expresses a theoretical relation that holds only for the special case in which each click is equally effective, there is no such constraint on the empirical relation described by Equation 4, which deals with the actual effect of the train. Comparison between the optimal and the obtained functions produces the relation between $N_{n}$ and $n$, which, when combined with the "method of subtraction," shows the relative effectiveness of each click in producing the detection. From the joint relation, $N_{n}=$ $f(n, I C I)$, one may determine the extent to which the relatively poor performance observed at high rates is due to a lack of information transduced after the initial transient.

\section{Nonlinearity in the Binaural \\ Response to High Rates}

One can imagine a variety of neural processes that might curtail the use of information derived from trains with high rates. Hafter and Dye (1983) showed that many of these processes affect the relations described by Equation 4 in essentially the same way. This group includes (1) neural refractorinesses, which make it possible for a click to occur during the refractory periods of individual units; (2) the restriction of bandwidth imposed by the auditory filters, which reduces the depth of modulation and thus increases variance in the transduction from mechanical to neural activity; and (3) any lack of independence between successive samples of the added internal noise. Although each of these processes is informationdestructive, albeit for different reasons, they all predict that the result of higher click rates should be to reduce performance in such a way that the terminal slope of the $\log$ threshold, $\log n$ functions remain a constant -0.5 . Because a slope of -0.5 means that $N_{n}$ is proportional to $n$, the effects of the three limiting processes are said to be stationary; that is, they act uniformly throughout the duration of the train.
The most interesting fact to come out of the experiments on lateralization has been that stationarity does not hold for higher click rates. Numerous experiments (Buell \& Hafter, 1986; Dye \& Hafter, 1984; Hafter \& Buell, 1985; Hafter \& Dye, 1983; Hafter, Dye, \& Wenzel, 1984) have shown that for fairly low rates (ICIs on the order of 10 to $12 \mathrm{msec}$ ), the slopes of the $\log$-log functions are indeed 0.5 . However, this is not the case for higher rates, where the data are better fit by the expression

$$
\log \Delta \mathrm{IDT}_{n}=\log \Delta \mathrm{IDT}_{1}-0.5 k \log n,
$$

where $0 \leq k \leq 1$ and $k=f(\mathrm{ICI})$. Equation 5 captures the experimental fact that the slopes grow more shallow with increased rate, the limiting case of 0.0 having been observed for ICIs on the order of $2 \mathrm{msec}$. The change in slope shows that, rather than the usually considered limitations on high stimulus rates ( 1 through 3 above), there is a process whose action is nonuniform over the duration of the stimulus. By applying the logic of Equation 4 to the inverse logarithm of Equation 5,

$$
\Delta \mathrm{IDT}_{n}=\frac{\Delta \mathrm{IDT}_{1}}{n^{(k)(0.5)}},
$$

we find that the relation between numbers of clicks and evoked neural responses is a compressive power function,

$$
N_{n} \propto n^{k},
$$

where $0 \leq k \leq 1$. Values of $k$ less than 1.0 indicate that each click in the train evoked fewer responses than the one that preceded it.

The hypothetical results shown in Figure 1 illustrate this interpretation for conditions distinguished by ICIs that range from relatively long at the top to relatively short at the bottom. The first column plots log thresholds against $\log n$ for $k \mathrm{~s}$ of $1.0,0.7$, and 0.2 , and so slopes of -0.5 , $-0.35,-0.1$, respectively. The second column shows the relation between $N_{n}$ and $n$ implied by Equation 7. The vertical bars of the third column represent the derivatives of the functions in column 2 at discrete values of $n$. These represent the relative effectiveness of each click in the train. Note that for the stationary case, $k=1$, all clicks are equally effective, whereas for $k=.2$, virtually no information is transduced beyond the first. In earlier papers, we referred to this as "post-onset saturation"; however, because saturation has multiple meanings, we have adopted the name "binaural adaptation" (Hafter et al., in press). A review of much of the research on binaural adaptation can be found in Hafter and Buell (1985).

\section{Are There Rate-Dependent Changes in the Discrimination of Periodicity?}

Hafter and his colleagues (Hafter, 1983; Hafter \& Buell, 1985; Hafter et al., in press; Hafter \& Wenzel, 1987) have provided evidence to suggest that the neural locus of "binaural adaptation"' is at a level in the auditory system peripheral to binaural interaction, in what are essentially monaural channels. Thus, it was natural to examine the 

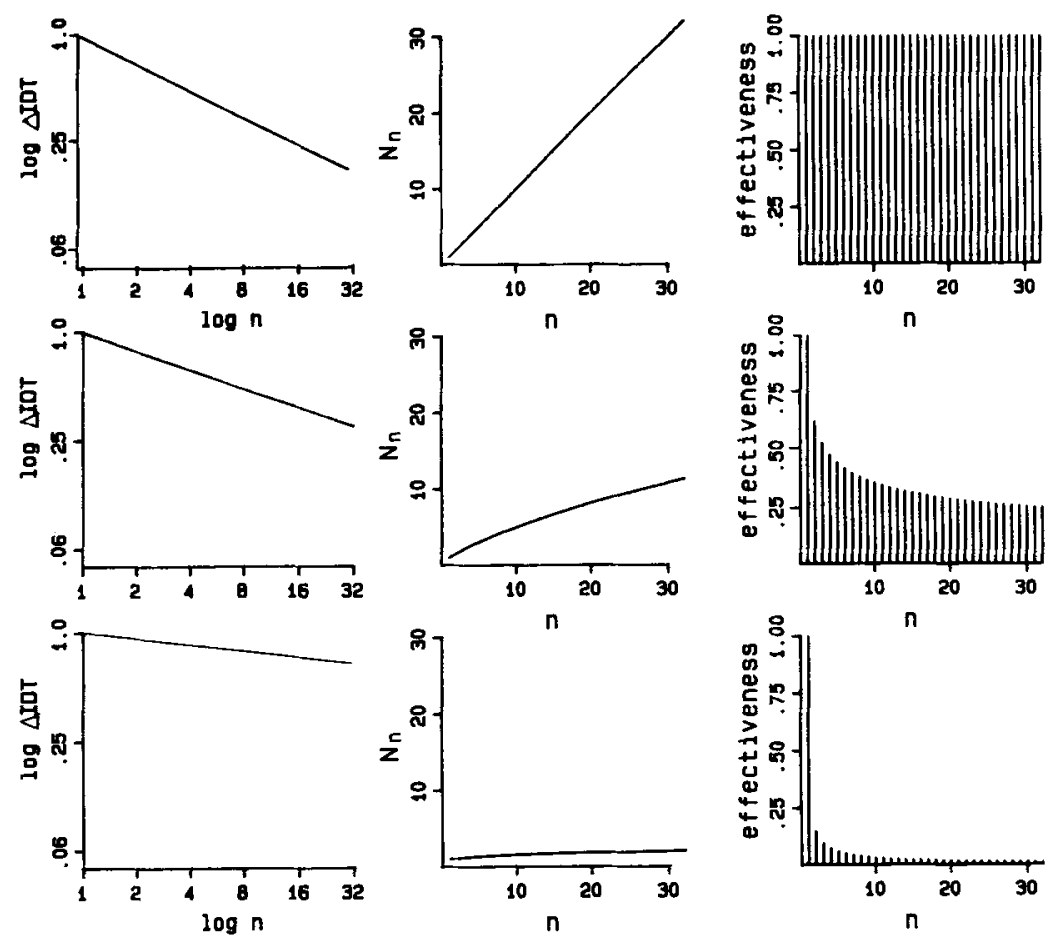

Figure 1. Hypothetical lateralization results and interpretation. The first column presents hypothetical lateralization data (based on Hafter \& Dye, 1983) as the interclick interval (ICI) is reduced (top to bottom). Thresholds for interaural differences in time ( $\triangle I D T)$ are plotted as a function of the number of clicks in the train $(n)$. As the click rate is increased, the slope of the function relating $\log \Delta \mathrm{IDT}$ and $\log n$ is reduced. The second column presents the implied relationship between the hypothetical variable $N_{n}$ and the number of clicks in the train. $k \mathrm{~s}$ of $1.0,0.7$, and 0.2 were used. The third column presents the effectiveness of each click in the train. For long ICIs, each click is equally informative; for short ICIs, little information is transduced beyond the first click.

joint effects of envelope rate and duration in a monaural task, namely, frequency discrimination. The stimuli were similar to those used in the binaural experiments, except that the cues to be detected were changes in the click rate (CR). Following the logic described above, one may assume that the task is limited by an internal noise (temporal jitter) added to each of the $(n-1)$ periods in the click train. Calling the number of periods $m$, then, for trains of lengths $m_{i}$ and $m_{j}$, the situation in which each period is equally effective provides the relationship

$$
\Delta \mathrm{CR}_{m_{j}}=\frac{\Delta \mathrm{CR}_{m_{i}}}{\left\{m_{j} / m_{i}\right\}^{0.5}} .
$$

Similarly, its logarithmic form is a straight line with a slope of -0.5 :

$$
\log \left(\Delta \mathrm{CR}_{m_{j}}\right)=\log \left(\Delta \mathrm{CR}_{m_{i}}\right)-0.5 \log \left\{m_{j} / m_{i}\right\}
$$

Since the point of the experiment was to examine the relative effectiveness of each period, we have again resorted to a hypothetical intervening variable, $M_{m}$, here defined as the number of neural representations of the $m_{m}$ periods. As before, the efficacy of successive portions of the envelope would be defined by the relation between
$M_{m}$ and $m$, found by comparison of data with the hypothetical function

$$
\log \left(\Delta \mathrm{CR}_{m_{j}}\right)=\log \left(\Delta C R_{m_{i}}\right)-0.5 \log \left\{M_{m_{j}} / M_{m_{i}}\right\}
$$

\section{METHOD}

In a two-interval, forced-choice (2IFC) procedure, listeners compared diotic trains whose CRs were $1 / \mathrm{ICI}$ and $1 /(\mathrm{ICI}+\Delta \mathrm{ICI}) \mathrm{Hz}$, respectively. Although their spectra consisted of predominantly higher harmonics, these trains have a distinct pitch, similar to those of tones whose frequencies equal the corresponding fundamentals.

\section{Spectra}

Figure 2 describes the stimulus, in both the time and frequency domains. Figure 2 a shows the building block for a train, a single click. It was produced by multiplying a Gaussian envelope by a sinusoid, the center frequency $\left(f_{\text {cen }}\right)$, which was set to cosine phase at the center of the envelope. The amplitude spectrum of such a click is continuous and Gaussian, and centered at $f_{\text {cen }}$. Figure $2 \mathrm{~b}$ depicts a hypothetical train whose CR is $250 \mathrm{~Hz}$ but whose length is infunite. It has a line spectrum with components only at harmonics of the fundamental frequency. The amplitudes of the components are weighted by the same Gaussian spectrum as that of a single click. Figure $2 c$ shows the result of fixing the length of the train. Here, 


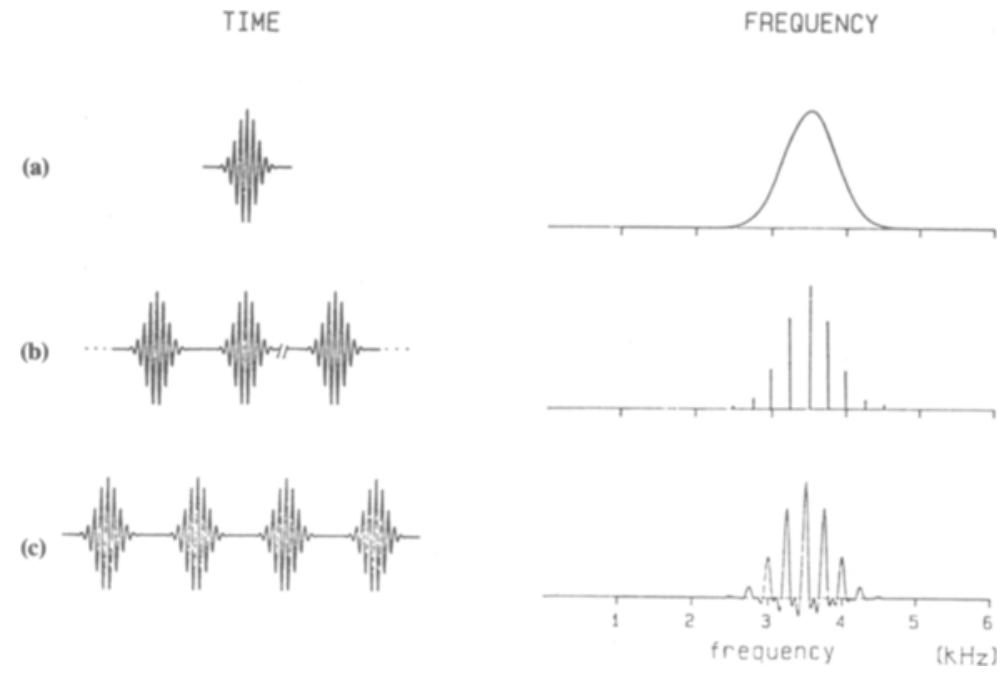

Figure 2. Time and frequency representation of the signal. The top panel (a) shows a single Gaussian click (center frequency of $3500 \mathrm{~Hz}$ ). The middle panel (b) presents a hypothetical, infinitely long click train, with a 4-msec ICI (250-Hz click rate). The bottom panel (c) presents a train of four clicks.

energy is spread into regions between the harmonic frequencies according to the convolution of the line spectrum of Figure $2 \mathrm{~b}$ and a $\sin c$ function, $\sin (\pi \cdot f \cdot D) / \pi \cdot f \cdot D$, where the duration $(D)$ consists of $m$ periods plus the width of the terminating click.

\section{Center Frequencies and Bandwidths}

Two different bandwidths, determined by the durations of the individual clicks, were tested. How one describes the duration of a Gaussian impulse is somewhat arbitrary; we used the standard deviation of the Gaussian envelope. Similarly, bandwidth is described by the distance in the amplitude spectrum between components at $f_{\text {cen }} \pm \sigma_{f} \mathrm{~Hz}$. Table 1 presents parameters of clicks in the nominally narrow- and wideband conditions.

Signals were created with a PDP-11 computer using a sampling rate of $50 \mathrm{kHz}$. The digital signals were then transformed via a 14bit digital-to-analog converter with deglitching and passed through a 48-dB/octave analog filter (Frequency Devices 901F) set to $10 \mathrm{kHz}$. The signal level was set such that a train of clicks presented at $100 \mathrm{clicks} / \mathrm{sec}$ had an RMS sound pressure level of $60 \mathrm{~dB}$ SPL. Given normal listening abilities, only the central portion of the spectrum and the two or three lobes on either side of it were assumed to be audible in the narrow-band conditions; the wideband conditions offered an increased number of potentially audible components but at the expense of reducing the absolute levels of common components.

For most of the conditions tested, the center frequency was $3500 \mathrm{~Hz}$ (see below for a discussion of cases in which the center frequency was varied within a trial). The values of $C R$ ranged from

Table 1

Durations and Bandwidths for the Two Types of Clicks

\begin{tabular}{lcc}
\hline Click Type & $\begin{array}{c}\text { Duration } \\
(\mathrm{msec})\end{array}$ & $\begin{array}{c}\text { Bandwidth } \\
(\mathrm{Hz})\end{array}$ \\
\hline Narrow band & 0.45 & 700 \\
Wideband & 0.28 & 1120 \\
\hline
\end{tabular}

Note-Clicks were made by multiplying a Gaussian weighting function by a cosine. Duration here refers to the standard deviation of the Gaussian envelope. The amplitude spectrum of such trains of clicks is also Gaussian, and the bandwidths given here refer to frequencies included between $\pm 1 \sigma$ points.
150 to 400 clicks/sec. Over the course of the experiment, the lengths of trains ranged from 8 to 64 clicks.

\section{The Roving Center Frequency}

Although all of our subjects claimed to hear the fundamental pitches (later confirmed by pitch matching with an oscillator), and to base their responses on them, we thought it necessary to consider the possibility that the listener might treat the experiment as a problem in amplitude discrimination, monitoring the levels in an auditory filter at the frequency of one of the components and saying "'low pitch" or "high," depending on those levels (Henning, 1967). Although some investigators (e.g., Viemeister \& Burns, 1976) have attacked this problem by generating periodicity with modulated noise, with pitch generated from repeated pulses, another solution was needed. For this reason, a subset of the experiment was repeated, using a roving center frequency. In these cases, $f_{\text {cen }}$ for each interval of the 2 IFC was chosen at random from a list ranging uniformly from 3000 to $3500 \mathrm{~Hz}$. Thus, within any given auditory filter, the interval containing the higher level could come from either the lower or higher click rate. As shown in Figure 2, the individual components were always a harmonic sequence of the CR, regardless of the center frequency of the Gaussian filter. Thus, roving $f_{\text {cen }}$ affected only the relative amplitudes of the sequence.

Two CRs, 250 and $167 \mathrm{~Hz}$, and four train lengths $(m), 7,15$, 31 , and 63, were tested. Thresholds for these conditions were completed using both roving and fixed center frequencies.

\section{Lowpass Noise}

When studying the presumed effectiveness of the envelope of a complex signal, one must worry about potential confounds introduced by nonlinear distortion in either the apparatus or the auditory systems of the listeners. To check for this possibility, a subset of the conditions were tested both in the quiet and in the presence of a lowpass noise. When present, continuous noise was shaped by two Krohn-Hite filters (3500R and 3100) placed in tandem; together, these produced an attenuation on the lowpass skirt of $48 \mathrm{~dB} /$ octave. The 3-dB downpoint was at approximately $2000 \mathrm{~Hz}$. The spectral density of the lowpass noise was $0 \mathrm{~dB}$ SPL.

Thresholds were determined both with and without lowpass noise for CRs of 150,250 , and $400 \mathrm{~Hz}$. The durations of the trains, in units of $m$, ranged from 7 to 47 . 


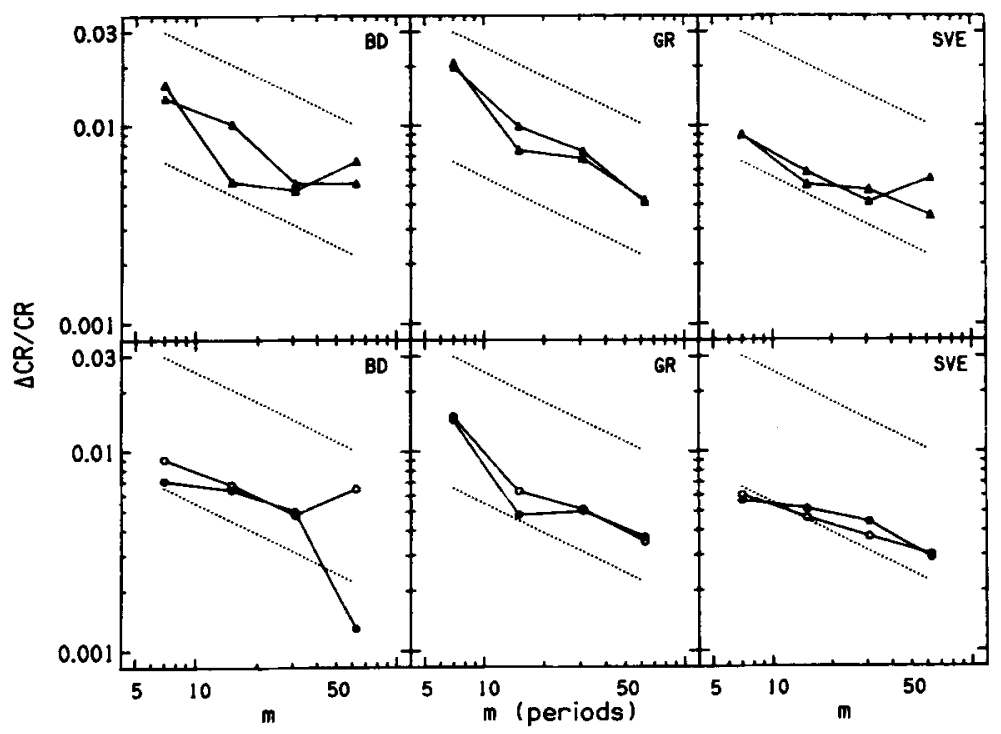

Figure 3. Thresholds for changes in click rate $(\triangle C R / C R)$ as a function of the number of periods in the click train $(m)$. Both are plotted logarithmically. The top panel presents data for a CR of $167 \mathrm{~Hz}$ (triangles), the bottom panel a CR of $250 \mathrm{~Hz}$ (circles). Filled symbols indicate that the center frequency was roved $(3000-3500 \mathrm{~Hz})$; open symbols indicate a fixed center frequency $(3500 \mathrm{~Hz})$. The dashed lines have a slope of $-\mathbf{0 . 5}$. Data for 3 subjects are presented.

\section{Procedure}

Within the 2IFC, each trial consisted of the two intervals to be discriminated, separated by $300 \mathrm{msec}$, plus approximately $2 \mathrm{sec}$ for a response. The subjects used buttons to indicate which of the two pitches was higher, and they received immediate visual feedback. Percentages of correct responses were computed for runs of 50 trials and used to draw psychometric functions based on at least eight runs per function. The interpolated point for $76 \%$ correct served to define thresholds (Elliot, 1964). Each subject had at least $40 \mathrm{~h}$ of experience at discriminating pitches in complex signals before data collection began. In general, the data for a given $C R$ were gathered in a block, with the order of testing being different across subjects. For each such block, the values of $m$ to be tested were presented in a random order.
Because it is often difficult, if not impossible, to maintain contact with student subjects throughout the course of a long experiment, only 1 subject, G.R. (one of the authors), served in all conditions. Three of the other 4 listeners were paid undergraduates and 1, B.D., was a graduate student of composition in the Department of Music at the University of California.

\section{RESULTS}

Because there are so few data on the pitches of trains of high-frequency clicks (an exception being those from Hoekstra, 1979), we felt it necessary to control for the possibility that judgments would be based on information

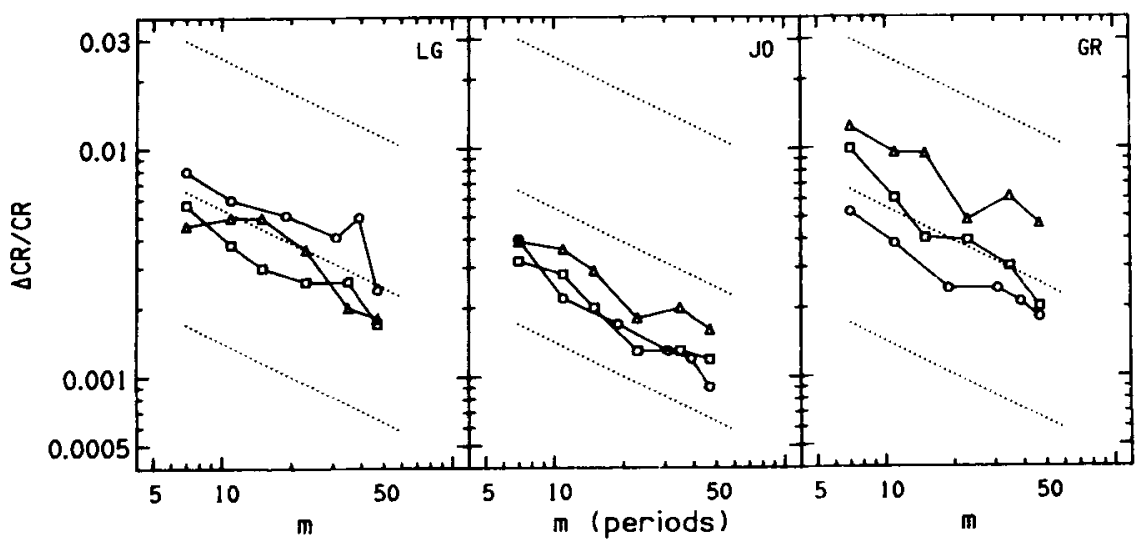

Figure 4. Thresholds for changes in click rate $(\triangle C R / C R)$ as a function of the number of periods in the click train $(m)$. Both are plotted logarithmically. Three click rates are presented, $150 \mathrm{~Hz}$ (triangles), $250 \mathrm{~Hz}$ (circles), and $400 \mathrm{~Hz}$ (squares). The center frequency was fixed at $3500 \mathrm{~Hz}$, and the data were collected in quiet. To aid in comparison, the top two dashed lines are as in Figure 3. Data for 3 subjects are presented. 


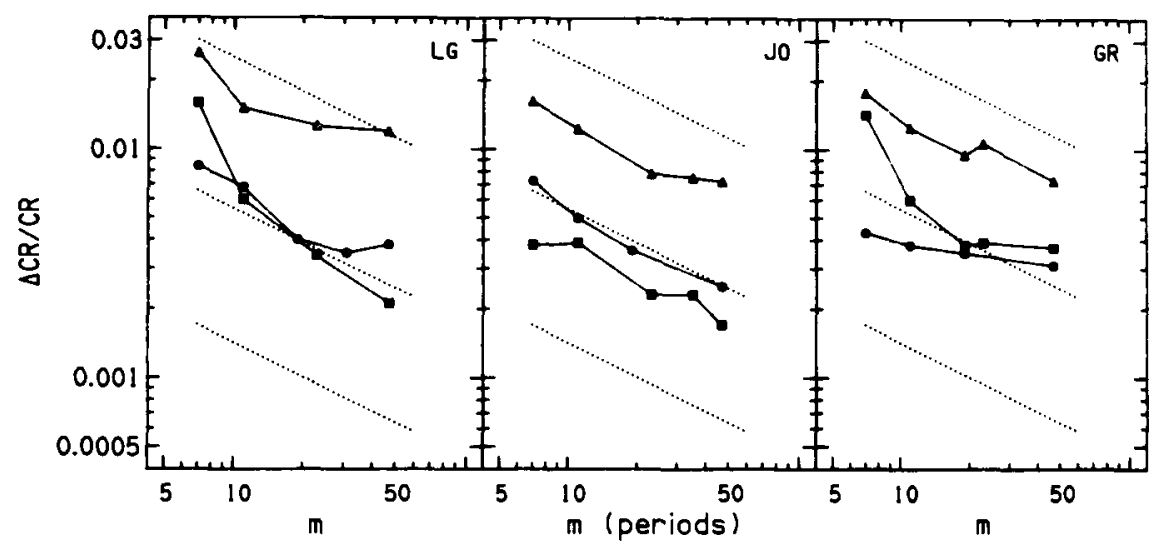

Figure 5. Thresholds for changes in click rate $(\triangle C R / C R)$ as a function of the number of periods in the click train $(m)$. As Figure 4, except that lowpass noise was continuously present.

other than the envelope's periodicity. Before examining the issue of primary concern, the effect of duration on the discrimination of period, we will show that the use of a roving center frequency and the addition of lowfrequency noise were unnecessary precautions.

Figure 3 shows comparisons between conditions in which the center frequency was either fixed or roving. Only wideband (short-duration) clicks were used. Data for the two CRs, 167 (top) and 250 (bottom) clicks/sec, are drawn separately. The three panels present data from 3 listeners. Open points are from conditions in which the center frequency was fixed at $3500 \mathrm{~Hz}$; closed points are from cases in which it was allowed to rove. The dotted lines indicate a slope of -0.5 . The ordinate is a logarithmic plot of the Weber fraction, $\triangle \mathrm{CR} / \mathrm{CR}$; the abscissa is a logarithmic plot of the number of periods in a train, $m$. Except for producing especially high variability in the performance of Subject B.D., there was no apparent effect of roving the center frequency; differences between these two conditions were nonsignificant $[F(2,4)=0.24]$. More importantly, there was no interaction between CR and the number of periods $[F(3,6)=2.98]$, with the functions relating $\log \Delta C R$ and $\log m$ being reasonably linear, with average slopes of about -0.5 (Table 2 , upper panel). This differs from lateralization results in which slopes of the $\log$ threshold, $\log n$ functions depend on rate.

Figures 4 and 5 show the effects of introducing lowpass noise. Here, the range of rates tested was expanded to include 150,250 , and 400 clicks $/ \mathrm{sec}$, with the latter requiring the use of clicks of shorter durations and hence wider bandwidths. Figure 4 presents the data obtained in quiet; Figure 5 presents those collected in the presence of lowpass noise. The parameter is CR, with triangles, circles, and squares indicating rates of 150,250 , and $400 \mathrm{clicks} / \mathrm{sec}$, respectively. Again, the dotted lines indicate a slope of -0.5 . While it seems that the absolute values of the thresholds were slightly elevated by the noise, particularly when the CR was $150 \mathrm{~Hz}$, these changes were nonsignificant at the .05 level $[F(1,2)=$ 12.07]. This is consistent with Hoekstra's (1979) obser- vation that lowpass noise did not affect the relationship between the discriminability of period and the center frequency of the signal.

Again, there was no apparent interaction between $\log \Delta \mathrm{CR}$ and $\log m[F(4,8)=1.38]$. This lends credibility to the conclusion that the functions are reasonably linear with slopes of close to -0.5 (Table 2 , lower panel).

\section{DISCUSSION}

\section{Effects of Roving the Center Frequency}

When we first listened to these stimuli, we were struck with the strong sense of pitch they produced, despite the fact that most of the energy was concentrated at rather high harmonics. We have found this to be true of trains of Gaussian pulses, even when the center frequencies are much higher than the ones used here (albeit, with increasingly higher harmonic numbers, there is a decrease in the frequency of tones set to match the pitch of the complex; Terhardt, 1971; Wallicer, 1969). Indeed, the pitch remains strong even when lowpass noise was present in the "dominance region" for pitch (Plomp, 1967; Ritsma, 1967). Although we have not examined pitch strength directly, we are inclined to think that the suprisingly strong pitch is due to either the Gaussian filter shape or the relatively wide bandwidth used (Wightman, 1973). Because of the pitch salience, we were not surprised when the roving $f_{\text {cen }}$ turned out to have little effect, strengthening our conviction that listeners were attending to periodicities rather than discriminating matched components as discussed in the Method section.

\section{Effects of the Bandwidths (Durations) of the Individual Clicks}

Wideband clicks were used here solely because their shorter durations would allow for testing at 400 clicks/sec. Because of their relatively late introduction into the experiment, no direct comparisons across bandwidths can be made. However, one should note that performance tended to be better with the wideband clicks, a finding 
Table 2

Slopes of the Log-Relative Threshold, Log $m$ Functions for the Longer Click Trains

\begin{tabular}{cccccccc} 
& & LP & Center & Click & \multicolumn{5}{c}{ Subjects } \\
\cline { 5 - 7 } BW & Noise & Freq & Rate & B.D. & S.V.E. & G.R. & Averages \\
\hline Narrow & no & Fixed & 167 & $-.39(.43)$ & $-.40(.90)$ & $-.67(.89)$ & $-.51(.81)$ \\
Narrow & no & Fixed & 250 & $-.19(.46)$ & $-.31(1.0)$ & $-.63(.93)$ & $-.42(.92)$ \\
Narrow & no & Roved & 167 & $-.50(.90)$ & $-.26(.56)$ & $-.69(.99)$ & $-.53(.97)$ \\
Narrow & no & Roved & 250 & $-.72(.75)$ & $-.29(.88)$ & $-.56(.77)$ & $-.56(.90)$ \\
& & & & & & & \\
& & & & & Subjects & & \\
Wide & no & Fixed & 150 & $-.50(.90)$ & $-.58(.80)$ & $-.52(.83)$ & $-.53(.94)$ \\
Wide & no & Fixed & 250 & $-.69(.96)$ & $-.46(.74)$ & $-.52(.94)$ & $-.56(.92)$ \\
Wide & no & Fixed & 400 & $-.57(.92)$ & $-.54(.91)$ & $-.75(.94)$ & $-.63(.96)$ \\
Wide & yes & Fixed & 150 & $-.44(.92)$ & $-.38(.76)$ & $-.43(.96)$ & $-.42(.93)$ \\
Wide & yes & Fixed & 250 & $-.55(.97)$ & $-.44(.83)$ & $-.17(.97)$ & $-.41(.94)$ \\
Wide & yes & Fixed & 400 & $-.47(.94)$ & $-1.0(.93)$ & $-.66(.79)$ & $-.77(.93)$ \\
\hline
\end{tabular}

Note-Individual subject and averaged data are shown. Relative thresholds are the obtained threshold (for each subject and condition) divided by the threshold for that subject and condition found with the largest $m$. Values of the squared correlation coefficients are indicated in parentheses. $B W=$ bandwidth, $L P=$ lowpass.

consistent with results both in lateralization (Wenzel, 1984) and in rate-discrimination (Richards \& Hafter, 1983).

\section{Effects of the Lowpass Noise}

Although nonsignificant, there appeared to be a slight rise in the discrimination thresholds due to the presence of the lowpass noise. If so, one could argue that the noise interfered with effective usage of the lowest components in the signals. Considered in conjunction with the special importance attributed to lower harmonic numbers in the determination of pitch (e.g., Plomp, 1967; Ritsma, 1967), it might further lead to speculation that our subjects applied heavier weightings to the lower harmonics, even though those amplitudes were relatively small. Nevertheless, it is important to note that while discriminability was marginally reduced when lowpass noise was present, there was no effect on the relationship between log threshold and $\log m$.

\section{Effects of Click Rate on the Absolute Thresholds}

In Figures 4 and 5, we see that although the differences between thresholds at 250 and 400 clicks/sec were not large, there was a marked reduction in performance at the lower rates. Decreasing the CR decreases the spacing of the spectral components, and there are at least two ways in which this might affect discrimination. For one, it has been suggested that the pitch derived from higher harmonic numbers is simply less salient (Goldstein, 1973). Second, with the level of each individual click set to $40 \mathrm{~dB}$ SPL, each of the more densely packed spectral components would, of necessity, be less intense. Unfortunately, the importance of pitch salience for discrimination is impossible to test directly, and, because we used only one signal level, we cannot test the importance of the individual component intensities.

\section{Effects of Increasing Duration}

An appealing feature of the log threshold, log number function is that its slope reflects the efficiency with which information is transmitted throughout the click train, regardless of the stimulus parameter being tested. Thus, we have compared the efficiency with which information is extracted across such different stimuli as interaural differences of time and level. Table 2 shows the least squares fitted slopes to all of the conditions tested. Squared correlation coefficients $\left(r^{2}\right)$ are indicated in parentheses. In order to visualize the comparison of slopes across conditions, data from Figures 3, 4, and 5 were replotted as relative thresholds, defined as the ratio of the actual value of each $\triangle C R / C R$ divided by the $\triangle C R / C R$ found for the largest number of periods tested for that particular condition. The scaling of performance relative to that with the largest $m$ was done separately for each listener and condition before averaging. By parsing individual differ-

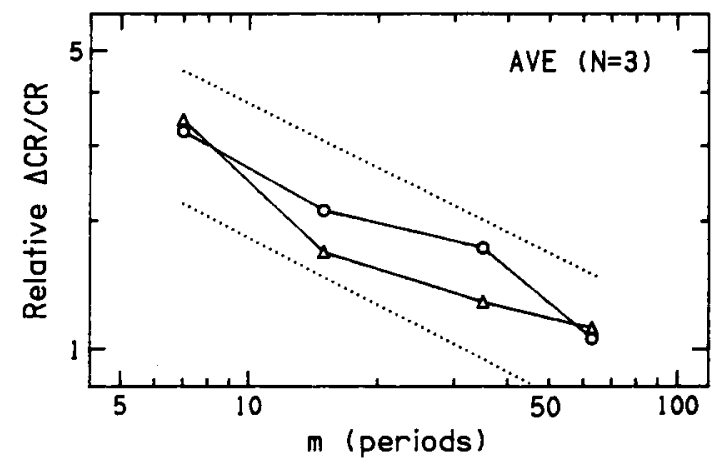

Figure 6. Average relative thresholds are indicated as a function of the number of periods for click rates of $167 \mathrm{~Hz}$ (triangles) and $250 \mathrm{~Hz}$ (circles). The values are based on the average of 3 subjects and both center frequency conditions (fixed and roved; Figure 3). 


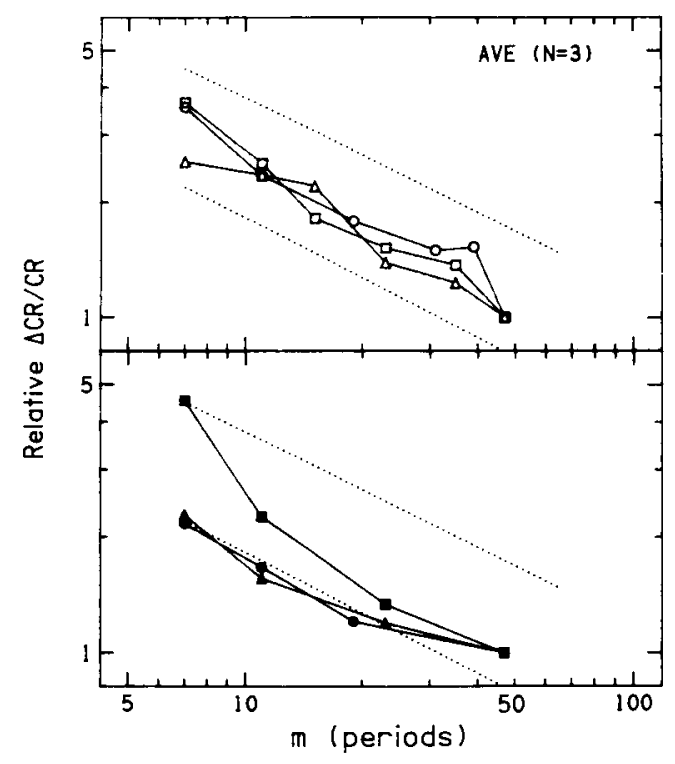

Figure 7. Average relative thresholds are indicated as a function of the number of periods for click rates of $150 \mathrm{~Hz}$ (triangles), $250 \mathrm{~Hz}$ (circles), and $400 \mathrm{~Hz}$ (squares). Open symbols indicate that thresholds were obtained in quiet (top panel, derived from Figure 4), and closed symbols indicate the presence of lowpass noise (bottom panel, derived from Figure 5).

ences in sensitivity, comparison across subjects is facilitated. Figure 6 is based on the data from Figure 3, averaged across the fixed and roving $f_{\text {cen }}$ conditions and averaged across all subjects. Figure 7 results from Figures 4 and 5, preserving the distinction between those obtained with (bottom panel) and without (top panel) lowpass noise.

When comparing performance with signals of various lengths, one must consider at least two sources of variance. The first might be thought of as decision variance; it is added subsequent to analysis in the sensory channels, and so is unaffected by duration. For shorter durations, it generally is quite small relative to variation in the sensorineural code, and thus of little influence. Here one would expect to see its presence only with long durations where it sets the floor in performance. The more important factor for perceptual processing is the sensory noise added to the neural code. This source of variance should be reduced by successive sampling of the stimulus information so that the rate by which performance grows with increasing signal duration can be used to infer the effectiveness with which successive portions of the signal are transduced into neural code. With this in mind, we note that despite variability across subjects, it is clear that the averaged slopes are close to the value of -0.5 ; this signifies that rate discrimination was based on information extracted uniformly from throughout the duration of the signal (Equation 10).

\section{Comparisons With Past Research}

Were the signal durations to increase without bound, an asymptotic performance floor would undoubtedly have been reached. The current data do not allow an unequivocal estimate of the point at which such a floor is reached, nor are we able to determine whether the asymptote is dependent on the signal duration versus the number of periods presented. We may, however, posit a minimum estimate of 50 clicks, or $330 \mathrm{msec}$ (on the basis of a CR of $150 \mathrm{~Hz}$ ). In contrast, both Yost (1980) and Buunen (1980), who studied the interaction of signal duration and pitch strength for ripple noise, demonstrated that, independent of the time delay employed, pitch strength fails to grow as the signal duration exceeds about 200 msec. Furthermore, the relative threshold for the discrimination of changes in the time delay of ripple noise is about 0.03 (Yost, Hill, \& Perez-Falcon, 1978), whereas the terminal value obtained here is about 0.002 . It is worth noting that there are at least two key differences between our stimuli and ripple noise stimuli. First, our stimuli are periodic (and deterministic). Second, our stimuli are relatively narrow band. The relative importance of these two aspects is unknown, and either could effect a change in the mechanism recruited for the discrimination task.

In an experiment similar to ours, Hoekstra (1979) examined the discriminability of changes in the rate of filtered click trains (1/3 octave bandpass filtered, $600 \mathrm{msec} \mathrm{du}-$ ration). For a CR of 200 clicks $/ \mathrm{sec}$, relative thresholds ( $70 \%$ correct discrimination) of approximately 0.001 and 0.015 were obtained for filters centered at 2000 and $4000 \mathrm{~Hz}$, respectively. Our terminal estimate of 0.002 ( $75 \%$ correct discrimination) is comparable.

Finally, we shall compare our thresholds with the values obtained by Moore (1973), who determined the jnd (75\% correct) for pure tones of varying durations. The average relative threshold for a $250-\mathrm{Hz}$ tone presented for $40 \mathrm{msec}(m=10)$ was 0.006 . For signal durations of $200 \mathrm{msec}(m=50)$, the average relative threshold was 0.003 . In the current experiment, "wide" clicks presented at a rate of 250 clicks/sec yielded average relative thresholds of 0.004 and 0.0017 for signal durations of 48 and $192 \mathrm{msec}$, respectively. Thus, our data and Moore's tonal data are reasonably close in both magnitude and the effect of signal duration on the threshold (on a log duration vs. $\log$ threshold plot, Moore's data yield a slope of $-0.43)$. For $2000-\mathrm{Hz}$ tones $(1500 \mathrm{~Hz}$ lower than the center frequency of the clicks used in the current experiment), Moore obtained average jnds of about 5.5 and $4.5 \mathrm{~Hz}$ for signal durations of 40 and $200 \mathrm{msec}$, respectively (a slope of -0.13 on a log duration, $\log$ jnd plot). Four-thousand-hertz tones led to average jnds of 18.9 and $14.6 \mathrm{~Hz}$ for signal durations of 40 and $200 \mathrm{msec}$, respectively (a slope of -0.16 on a log threshold, log jnd plot). Thus, for discriminability, changes in signal duration affect high-frequency clicks and tones at the frequency of the (missing) fundamental similarly; and changes in the 
signal duration affect high-frequency clicks and highfrequency tones differently.

\section{Comparisons With Lateralization and a Speculation About Adapting and Nonadapting Channels}

As discussed in the introduction, for lateralization of trains of clicks, the consistent finding has been that for higher CRs, the listener relies increasingly on information coded in the signals' onsets. Additional evidence has suggested that the site of the "binaural adaptation" is in the auditory periphery, prior to binaural interaction (Hafter \& Buell, 1985; Hafter et al., in press), and one reason for the present experiment was to see if temporal processing that did not require binaural interaction would show a similar special reliance on the onset.

Direct comparisons between results from the two kinds of tasks are instructive. From lateralization, discrimination of interaural delays produced average slopes of the $\log \Delta \mathrm{IDT}, \log n$ plots of -0.38 for a rate of 200 clicks $/ \mathrm{sec}$ and -0.22 for a rate of 500 clicks $/ \mathrm{sec}$ (Hafter \& Dye, 1983); similarly, discrimination of interaural differences of level produced average slopes of the $\log \Delta \mathrm{IDL}, \log n$ plots of -0.40 for rates of 200 clicks $/ \mathrm{sec}$ and -0.29 for rates of 500 clicks $/ \mathrm{sec}$ (Hafter et al., 1984). This differs greatly from the results found here for discrimination of click rates where slopes of $\log \Delta \mathrm{CR}, \log m$ functions seem independent of click rate. Thus, it would appear that the information-reductive process responsible for "binaural adaptation," although thought to occur prior to binaural interaction, does not affect the discrimination of ratedependent pitch.

These comparisons raise an interesting possibility. Here we have a case where very similar stimuli, that is, trains of high-frequency clicks, are treated very differently, depending on what kind of information is being extracted from them. Suppose that there are two types of channels in the monaural periphery. Neurons of the first kind carry information to be used for spatial hearing. They are somewhat "phasic" in response to high rates of stimulation, passing on to the binaural nervous system interaural information contained primarily in the signal's onset. The others are more "tonic," sending forward information to be used for discrimination of pitch that is drawn from throughout the stimulus. According to this view, the pathways that carry the message "what is the sound" monitor the signal continuously, whereas those responsible for tracking its direction rely on information in its onset. The notion of an anatomical as well as a functional dichotomy in the early response to high-frequency envelopes is intriguing, but it will require direct studies of electrophysiology for confirmation or refutation.

\section{SUMMARY}

1. Studies of lateralization based on interaural differences in the envelopes of modulated high frequencies have shown that as the envelope rate increases, the lateraliza- tion threshold is based increasingly on information derived from the stimulus onset. We have called this process "binaural adaptation."

2. Because previous experiments suggested that the neural site of "binaural adaptation" is in the auditory periphery, we questioned whether extraction of information about the signal's pitch would show a comparable reliance on the onset. Thus, listeners were presented with stimuli similar to those used in lateralization experiments, namely trains of filtered clicks centered at high frequencies, only here, they were asked to discriminate between trains with slightly differing periods.

3. A battery of control conditions included the use of low-frequency noise to prevent use of artifactual cues due to nonlinear distortions and of a roving-center-frequency technique designed to prevent discriminations based on comparisons between the levels of individual harmonic frequencies. Neither precaution proved essential.

4. Of primary interest was the effect of click rate on the relationship between discrimination and signal duration since it is from such functions that one can infer the relative efficacy of each period in a train. The idea is that differences in performance between trains of differing lengths are due to the adoption of additional samples of the information into the sensory code. Accordingly, integration of information throughout the train should result in a square-root reduction in the combined internal noise and, hence, a square-root reduction in threshold with increased duration.

5. The primary result was that unlike the case with lateralization, the information extracted for rate discrimination grew uniformly with stimulus duration for all of the click rates tested. It is suggested that there may be separate channels used to carry information for spatial hearing and for discrimination of pitch.

\section{REFERENCES}

Blauert, J. (1983). Spatial hearing. Cambridge, MA: MIT Press. Buell, T. N., \& Hafter, E. R. (1986). Parametric study of onset effects in lateralization. Journal of the Acoustical Society of America, 80, S107(A).

BUUNEN, T. J. F. (1980). The effect of duration on the prominence of pitch. In G. van den Brink \& F. A. Bilsen (Eds.), Psychophysical, physiological and behavioral studies in hearing (pp. 374-377). Delft, Holland: Delft University Press.

DYE, R. H., JR., \& HAFTER, E. R. (1984). The effects of intensity on the detection of interaural differences of time in high-frequency trains of clicks. Joumal of the Acoustical Society of America, 75, 1593-1598.

ElliotT, P. B. (1964). Tables of $d^{\prime}$. In J. A. Swets (Ed.), Signal detection and recognition by human observers: Contemporary readings (pp. 651-684). New York: Wiley.

Goldstein, J. L. (1973). An optimal processor theory for the central formation of the pitch of complex tones. Journal of the Acoustical Society of America, 54, 1496-1516.

Hafter, E. R. (1983). Lateralization of complex sounds. Journal of the Acoustical Sociery of America, 73, S2(A).

HAFTER, E. R., \& BUELL, T. N. (1985). The importance of transients for maintaining the separation of signals in auditory space. In M. I. Posner \& O. M. Marin (Eds.), Attention and performance XI . Hillsdale, NJ: Erlbaum.

Hafter, E. R., Buell, T. N., \& Richards, V. M. (in press). Onset- 
coding in lateralization: Its form, site and function. In G. Edelman, $\mathbf{M}$. Cowan, \& E. Gall (Eds.), Functions of the auditory system. New York: Wiley.

HAFTER, E. R., \& DYE, R. H., JR. (1983). Detection of interaural differences of time in trains of high-frequency clicks as a function of interclick interval and number. Joumal of the Acoustical Society of America, 73, 644-651

HafTer, E. R., Dye, R. H., JR., \& Wenzel, E. M. (1984), Detection of interaural differences of intensity in trains of high-frequency clicks as a function of interclick interval and number. Joumal of the Acoustical Society of America, 73, 1708-1713.

Henning, G. B. (1967). A model of auditory discrimination and detection. Joumal of the Acoustical Society of America, 42, 1325-1334.

HoEksTrA, A. (1979). Frequency discrimination and frequency analysis in hearing. Academic thesis, University of Groningen, Holland.

MoORE, B. C. J. (1973). Frequency difference limens for short-duration tones. Joumal of the Acoustical Society of America, 54, 610-619.

Nuetzel, J. M., \& HAFTER, E. R. (1981). Discrimination of interaural delays in complex waveforms: Spectral effects. Journal of the Acoustical Society of America, 69, 112-118.

Plomp, R. (1967). Pitch of complex tones. Journal of the Acoustical Society of America, 41, 1526-1533.

RichaRDS, V. M., \& HAFreR, E. R. (1983). Effect of duration on pitch discrimination of complex signals. Journal of the Acoustical Society of America, 74, S70(A).

RrtsmA, R. J. (1967). Frequencies dominant in the perception of the pitch of complex sounds. Journal of the Acoustical Saciety of America, 42, 191-198.

Schouten, J. F. (1940). The residue and the mechanism of hearing. Koninklijke Nederlandse Akademie van Wetenschappen: Proceedings. 43, 991-999.

Schouten, J. F. (1970). The residue revisited. In R. Plomp \& G. F.
Smoorenberg (Eds.), Frequency analysis and periodicity detection in hearing (pp. 41-54). Leyden, Holland: Sijtoff.

Schouten, J. F., Ritsma, R. J., \& Cardozo, B. L. (1962). Pitch of the residue. Journal of the Acoustical Society of America, 34, 1418-1424.

Terhardi, E. (1971). Die Tonhohe harmonischer Klang und das Oktavintervall. Acoustica, 24, 125-136.

TERHARDT, E. (1974). Pitch, consonance and harmony. Journal of the Acoustical Society of America, 55, 1061-1069.

VIEMEISTER, N. F., \& BURNS, E. M. (1976). Non-spectral pitch. Joumal of the Acoustical Society of America, 60, 863-869.

WALLICER, K. (1969). Zusammenhang zwischen den Schallreiz und der Periodentonhohe. Acoustica, 21, 319-329.

WENZEL, E. M. (1984). Lateralization of high-frequency clicks based on interaural time: Additivity of information across frequencies. Unpublished doctoral dissertation, University of California, Berkeley. Wightman, F. L. (1973). The pattern-transformation model of pitch. Journal of the Acoustical Society of America, 54, 407-416.

YosT, W. A. (1980). Temporal properties of the pitch and pitch strength of ripple noise. In G. van den Brink \& F. A. Bilsen (Eds.), Psychophysical, physiological and behavioral studies in hearing (pp. 367373). Delft, Holland: Delft University Press.

Yost, W. A., \& HAFTER, E. R. (1987). Lateralization. In W. A. Yost \& G. Gourevitch (Eds.), Directional hearing (pp. 49-84). New York: Springer-Verlag.

Yost, W. A., Hill, R., \& Perez-Falcon, T. (1978). Pitch and pitch discrimination of broadband signals with rippled power spectra. Journal of the Acoustical Society of America, 63, 1166-1173.

(Manuscript received July 2, 1987; revision accepted for publication October 9,1987 .) 